\title{
Prolonged thrombocytopenia in a child with severe neonatal alloimmune reaction and Noonan syndrome
}

\author{
Inês Salva, Sara Batalha, Raquel Maia, \& Paula Kjollerstrom \\ Department of Pediatric Hematology, Hospital de Dona Estefânia, Centro Hospitalar de Lisboa Central, Lisbon, Portugal
}

\begin{abstract}
Fetomaternal alloimmune thrombocytopenia (FMAIT) caused by maternal antibodies is the leading cause of severe neonatal thrombocytopenia. A 1-month-old Caucasian girl was referred to our Hematology Clinic for persistent thrombocytopenia diagnosed after a bleeding episode. Diagnostic tests suggested FMAIT. Mild thrombocytopenia persisted for 18 months, and subsequent findings of dysmorphic facies, short stature and mild pulmonary stenosis led to the hypothesis of Noonan syndrome (NS), which was confirmed by genetic test. Other hematological abnormalities were excluded and she had no further bleeding episodes. This case illustrates the possibility of different diagnoses with the same clinical manifestations. The persistence of thrombocytopenia longer than expected associated with typical physical features led to the diagnosis of NS.
\end{abstract}

\author{
Keywords \\ Alloimmune, dysmorphism, neonatal, \\ Noonan syndrome, thrombocytopenia

\section{History} \\ Received 9 August 2015 \\ Revised 17 September 2015 \\ Accepted 1 October 2015 \\ Published online 24 November 2015
}

\section{Introduction}

Fetomaternal alloimmune thrombocytopenia (FMAIT) is the leading cause of severe neonatal thrombocytopenia and may cause intracranial hemorrhage (ICH) [1]. It is caused by transplacental transfer of maternal antibodies (anti-HPA), after maternal sensitization to paternally derived antigens, to fetal platelets, most commonly HPA-1a (in $75-90 \%$ of cases). Less common antibodies include anti-HPA-5b $(10-15 \%)[1,2]$. When ICH is absent, the prognosis is usually favorable and the platelet count typically recovers to normal values within 8 to 10 days of life [1,2]. For reasons not well understood, a low count sometimes persists for longer periods of time, occasionally for several months [3].

\section{Case report}

One month-old Caucasian girl, second child of a healthy $\mathrm{A} \mathrm{Rh}^{+}$ mother, presented to our outpatient clinic with a history of persistent thrombocytopenia.

The obstetric history was positive for mild maternal thrombocytopenia during the third trimester (Platelets $119,000 / \mu \mathrm{L}$ ). The mother was immune to toxoplasmosis and cytomegalovirus, and serologies were negative for other TORCH infections (including syphilis and rubella). The child was born at 37 weeks of gestation, with a birth weight of $3160 \mathrm{~g}$ and presented with umbilical cord hemorrhage at birth and multiple ecchymoses. Her physical exam was otherwise unremarkable. Blood tests revealed: hemoglobin $11.7 \mathrm{~g} / \mathrm{dL}$, reticulocyte count $8 \%$, leukocytes $16,130 / \mu \mathrm{L}$, platelets $14,000 / \mu \mathrm{L}$, and normal prothrombin time and partial thromboplastin time. During the first 2 weeks of life, platelet counts continued to decrease (minimum of $6000 / \mu \mathrm{L}$ on the fourth day of life) despite serial platelet transfusions (1st, 6th, 10th, 11th,

Correspondence: Sara Batalha, Hospital de Dona Estefânia, Rua Jacinta Marto, 1169-045 - Arroios, Lisboa, Portugal. Tel: 00351 213162200. E-mail: sara.batalha@gmail.com 12th, and 13th days of life) and administration of IV immunoglobulin $1 \mathrm{~g} / \mathrm{kg}$. Antiplatelet antibody HPA-3b was detected using enzyme-linked immunosorbent assay (ELISA).

The genetic study of the patient showed a $1 \mathrm{ab}, 2 \mathrm{aa}, 3 \mathrm{ab}, 5 \mathrm{ab}$, 4aa, 15ab genotype, while the mother's genotype was 1ab, 2aa, $3 \mathrm{aa}, 5 \mathrm{ab}, 4 \mathrm{aa}, 15 \mathrm{ab}$ and the father's genotype was 1ab, 2aa, 3ab, 5aa, 4aa, 15bb. In conclusion, the mother had HPA-3aa platelets, the child HPA-3b platelets, and the cross-match showed that the mother's serum was strongly reactive against the father's platelets. The child was diagnosed with FMAIT. Cranial ultrasound was negative for ICH and her echocardiogram showed only mild pulmonary stenosis.

On the third week of life, her platelet count started to increase to a maximum of $93,000 / \mu \mathrm{L}$ She required a red blood cell transfusion due to anemia (hemoglobin: $9.1 \mathrm{~g} / \mathrm{dL}$ ) on the 25 th day of life and was discharged home as she was clinically stable.

The child was referred to our outpatient clinic when she was 1 month old, due to mild and persistent thrombocytopenia despite the absence of episodes of hemorrhage or severe clinical manifestations. During follow-up in the first year of life (with platelet counts 48,000-110,000/ $\mu \mathrm{L}$ ), a dysmorphic facies (high forehead, low set ears, and micrognathia), failure to thrive, and short stature became increasingly apparent, leading to the hypothesis of Noonan syndrome (NS). A mutation in the PTPN11 gene c. $181 \mathrm{G}>\mathrm{A}$ in exon 3 was identified. Further tests for NS-renal ultrasound, thyroid function, coagulation factor assays (V, VII, VIII, IX, X, XI, XII, XIII, von Willebrand), and platelet aggregation as evaluated with PFA 100-revealed no other abnormalities.

She is now 19 months old and has had no further evidence of mucocutaneous bleeding.

\section{Conclusion}

NS is a relatively common congenital genetic disorder with an estimated prevalence of 1 in 1000 to 1 in 2500 live births [4]. Characteristic findings include distinctive facial features, short 
stature, chest deformity, and congenital heart disease, which may not be apparent during the first months of life and require a high index of suspicion during follow-up [5, 6]. It is an autosomal dominant disorder with complete penetrance but variable expressivity [3]. Mutations in PTPN11 occur in 50\% of patients, SOS1 in $13 \%$, RAF in $3-17 \%$, and KRAS in less than 5\% [7].

PTPN11 encodes SH2 domain, which contains protein-tyrosine phosphatases Shp1 and Shp2; Shp1 is expressed in hematopoietic and epithelial cells, while Shp2 is expressed widely and they are both key regulators in megakaryocyte development, platelet formation, and function (including interaction with collagen and fibrinogen); these two phosphatases seem to have opposite roles in platelet function (Shp1 reduces platelet response to collagen and fibrinogen by positively regulating immune receptor tyrosine-based activation motif-containing receptors and integrin signaling, whilst Shp2 behaves in the opposite way) [8].

Several hematological abnormalities have been detected in association with this syndrome including thrombocytopenia that may be severe; cases that resemble congenital amegakaryocytic thrombocytopenia have been described and may be caused by specific PTPN11 mutations (c.218C >T) [9, 10]. Although the mechanism responsible for this phenomenon is still incompletely understood, ineffective thrombopoiesis and spleen sequestration are two of the main hypotheses 11, 12]. Other hematological disorders include abnormal platelet function and deficiency of single or multiple coagulation factors (most commonly partial deficiency of factor XI) [4-7, 11-13].

Persistence of thrombocytopenia beyond the second week of life in a child diagnosed with FMAIT should prompt further investigation to exclude other etiologies. In this case, persistence of thrombocytopenia longer than expected associated with dysmorphic facies, pulmonic valve stenosis, developmental delay, and short stature led to the diagnosis of NS. The clinical presentation of severe thrombocytopenia at birth was probably due to the association between the immune mechanism and the congenital cause [14].

This case illustrates the fact that different diagnoses with the same clinical manifestations should be taken into consideration when expected evolution does not occur.

\section{Declaration of interest}

The authors report no declarations of interest.

\section{References}

1. Espinoza JP, Caradeux J, Norwitz ER, Illanes SE. Fetal and neonatal alloimmune thrombocytopenia. Rev Obstet Gynecol 2013;6(1):e15e21.

2. Kaplan C. Foetal and neonatal alloimmune thrombocytopaenia. Orphanet J Rare Dis 2006;1:39.

3. Peterson JA, McFarland JG, Curtis BR, Aster RH. Neonatal alloimmune thrombocytopenia: pathogenesis, diagnosis and management. Br J Haematol 2013;161(1):3-14. doi:10.1111/bjh.12235.

4. Romano AA, Allanson JE, Dahlgren J, Gelb BD, Hall B. Noonan syndrome: Clinical features, diagnosis and management guidelines. Pediatrics 2010;126(4):746-759.

5. Online Mendelian Inheritance in Man, OMIM®. MIM Number:163950. Baltimore, MD: Johns Hopkins University; 2013 Feb [cited 2015 Apr 27]; Available from: www.omim.org/entry/ 163950.

6. Sznajer Y, Keren B, Baumann C, Pereira S, Alberti C, Elion J, Cavé $\mathrm{H}$, Verloes A. The spectrum of cardiac anomalies in Noonan syndrome as a result of mutations in the PTPN11 gene. Pediatrics 2007;119:e1325-1331.

7. Allanson JE, Roberts AE. Noonan syndrome. In: Pagon RA, Adam MP, Ardinger HH, et al., editors. GeneReviews ${ }^{\circledR}$ (Internet). Seattle, WA: University of Washington, Seattle; 2011 Aug. 1993-2015 [cited 2015 Apr 28].

8. Mazharian A, Mori J, Wang Y, Heising S, Neel B, Watson SP, Senis YA. Megakaryocyte-specific deletion of the protein-tyrosine phosphatases Shp1 and Shp2 causes abnormal megakaryocyte development, platelet production and function. Blood 2013;120:4205-4220.

9. Christensen RD, Yaish HM, Leon EL, Sola-Visner MC, Agrawal PB. A de novo T73I mutation in PTPN11 in a neonate with severe and prolonged congenital thrombocytopenia and Noonan syndrome. Neonatology 2013;104:1-5.

10. Evans DG, Lonsdale RN, Patton MA. Cutaneous lymphangioma and amegakaryocytic thrombocytopenia in Noonan syndrome. Clin Genet 1991;39:228-232.

11. Flick JT, Singh AK, Kizer J, Lazarchick J. Platelet dysfunction in Noonan's syndrome. A case with platelet cyclooxygenase-like deficiency and chronic idiopathic thrombocytopenic purpura. Am J Clin Pathol 1991;95(5):739-742.

12. Briggs B, Dickerman JD. Bleeding disorders in Noonan syndrome. Pediatr Blood Cancer 2012;58:167-172.

13. Artoni A, Selicorni A, Passamonti SM, Lecchi A, Bucciarelli P, Cerutti M, Cianci P, Gianniello F, Martinelli I. Hemostatic abnormalities in Noonan syndrome. Pediatrics 2014;133(5):e1299-e1304.

14. Nunes P, Aguilar S, Prado SN, Palaré MJ, Ferrão A, Morais A. Severe congenital thrombocytopenia - First clinical manifestation of Noonan syndrome. BMJ Case Rep 2012; bcr1020114940. 
Copyright of Platelets is the property of Taylor \& Francis Ltd and its content may not be copied or emailed to multiple sites or posted to a listserv without the copyright holder's express written permission. However, users may print, download, or email articles for individual use. 